\title{
Anti-social Personality Disorder and Conduct Disorder (ASPD/CD), Ethnicity and other Characteristics of the Alcohol Treatment Population in Trinidad and Tobago \\ S Shafe, Shelley Moore
}

\begin{abstract}
Background: Individuals of East Indian and African ancestry constitute the largest population subgroups in Trinidad and Tobago. Many differences are observed in their drinking behaviour and are attributed to cultural and social factors. The aim of this paper is to determine if there are differences in personality disorder diagnosis in alcohol abuse/ dependent patients who attend treatment facilities in Trinidad and Tobago.
\end{abstract}

Methods: The data used in this study is from the Collaborative Group on the Genetics of Alcoholism (COGA) Trinidad and Tobago Sample. A total of 144 alcohol dependent individuals of East Indian and African ancestry were included in the study. Ethnicity was classified as having three grandparents from one of the two ethnic groups. A diagnosis of Antisocial Personality Disorder (ASPD) was determined using DSM-III-R, which not only confirms the presence this disorder and/ or conduct disorder before the age of 15 , but also identifies syndromal levels of anti-social behaviour after the age of 15 years. Patients with major medical problems that possibly impacted their drinking and were unrelated to alcohol dependence (e.g. cancer, severe heart or lung disease, diabetes etc.) were excluded. One hundred and thirteen (113) control subjects who were not alcohol dependent were matched by age, sex and ethnicity to one hundred and fourteen (144) alcohol dependent participants.

Keywords: Alcohol Abuse, Alcohol Dependence, Personality Disorder, Ethnicity.

From: ${ }^{1}$ Psychiatry Unit, Department of Clinical Medical Sciences, Faculty of Medical Sciences, University of the West Indies, St. Augustine, Trinidad and Tobago. 
Correspondence: Dr S Shafe, Psychiatry Unit, Department of Clinical Medical Sciences Faculty of Medical Sciences, Mount Hope, Trinidad and Tobago. Email: samuel.shafe@sta.uwi.edu

Result: This study did not identify any significant differences in personality disorder between the two ethnic groups in individuals with a diagnosis of alcohol dependence. There was a significant difference in anti-social personality disorder overall, between the alcohol dependent group and the control group. In this treatment sample, the percentage of East Indians with a diagnosis of ASPD was 7\% and of Africans was $11 \%$.

Conclusions: There were no significant differences $(p=0.383)$ in ASPD among alcohol dependent treatment subjects of East Indian and African ancestry in Trinidad and Tobago. However, this study was done in an environment with intense negative view about mental illness. This negative view may influence the type of information that participants provide about their drinking behaviour. Similarly, some behaviour may be socially acceptable, therefore accounting for low percentage of ASPD detected in the treatment group.

\section{BACKGROUND}

DSM-V (Diagnostic and Statistical Manual - version V) describes Antisocial Personality Disorder (ASPD) as being characterised by a pattern of disregard and violation of the rights of others with evidence from the age of 15 years (1). Arriving at this diagnosis requires clinical assessment that is centred on information collected from patients and third party sources. The accuracy of the assessment may be influenced by factors that include reporting practices and the perception of behavioural problems which may vary as a result of cultural practices. This 
is more so since the social acceptability of a particular behaviour may be rooted in cultural beliefs that can influence the type of information provided by informants. Another important factor is the ability of the participants to recollect events that happened many years ago (2). This review examines the relationship between ethnicity, alcoholism and ASPD among two major ethnic subgroups (East Indians and Africans) in Trinidad and Tobago. Trinidad and Tobago consist of two islands of the Caribbean with a population of 1.3 million, comprising individuals of East Indians (35.4\%), Africans (34.2\%) and mixed (22.8\%) ancestries (3).

Multiple studies reported the co-existence of substance use disorders and personality disorder (46) and other psychiatric disorders which is not the focus of this study. The occurrences are said to be particularly true for anti-social and borderline personality disorder $(7,8)$. In the United States (US) this co-occurrence is said to be pervasive in the general population, with a reported prevalence of 3.6\% for ASPD. Similarly, it was reported that the co-occurrence of ASPD and alcohol dependence was significantly greater $(p<0.006)$ for women than men $(7)$.

In the alcohol dependence treatment population, the rate of psychiatric co-morbidity is reportedly higher compared to the general population $(7,9,10)$. In a US study of hospitalised patients with a diagnosis of alcohol dependence (231 men and 90 women), ASPD was the most prevalent psychopathology in men (49\%) and in women the frequency was $20 \%$ (11). In contrast, there has been no reference to anti-social personality disorders among alcohol dependent groups in Trinidad and Tobago in past studies.

The aim of this paper is to contribute to the existing knowledge of the co-existence of anti-social personality disorder and alcohol dependence through investigation of ethnic subgroups in Trinidad and Tobago. 


\section{Ethnic variation}

In the US, co-morbidity of alcohol dependence and ASPD is reported to vary according to the subject's ethnicity (7). As part of the Collaborative Study of the Genetics of Alcoholism (COGA), data obtained of 240 Mexican American young adults in San Diego (alcohol dependent, $n=63$ and non-alcohol dependent, $n=177$ ), showed ASPD was co-morbid with alcohol dependence in women $(\mathrm{OR}=\mathbf{2 0 . 0}, p=0.006)$. However ASPD was not co-morbid in men and conduct disorder (CD) was not co-morbid with alcohol dependence in both sexes, with inadequate power blamed for this finding (7). In the Epidemiological Catchment Area study (ECA), a higher rate of alcoholism was reported for Hispanic Americans compared to Caucasians (10) and associated with length of stay in the US; co-morbid psychiatric disorders were considered as likely factors (8). However, a lower rate of psychiatric disorders was found

in Native American veterans compared with non-Native Americans diagnosed with alcohol and substance use disorders (12). An analysis of subjects, that included Caucasians (n=1177), African Americans (n=361), Hispanics (n=93) and Alaskans $(n=486)$, as part of COGA study noted significant differences in psychiatric co-morbidity. ASPD was more common among men than women across all ethnic groups and more common among Alaskan and Hispanic men than African-American and Caucasian men. Among women, ASPD was highest in Hispanics and lower rates reported among Caucasians and African-Americans (13).

In the Caribbean, ethnic differences related to drinking behaviour and hospital admission were reported in an unpublished paper (14) and these differences were explained by multiple factors including social, environmental and religious factors. In Trinidad and Tobago, ethnic differences were observed in previous studies with respect to alcohol consumption, clinical 
course of alcoholism as well as alcohol dependence, anxiety and affective disorder co-morbidity $(2,15)$. While it was noted that individuals of East Indian ancestry were more likely to present early for intervention compared to those of African ancestry, attitude and other cultural practices were said to play major roles in alcohol use practices. Hence determining causal associations of alcohol dependence and the prevalence of psychiatric disorders is very significant in order to address this issue within this population, where there is an estimated lifetime prevalence of alcohol use of $75-85 \%$ (16).

\section{METHODS}

\section{Subjects}

This report is based on data derived from the study of genetics of alcoholism obtained from three (3) Centres (i.e. Substance Abuse Prevention and Treatment Centres: Caura, San Fernando General and Scarborough General Hospitals) in Trinidad and Tobago. Approval was obtained from the Ethics Committee of the University of the West Indies, Ministry of Health of Trinidad and Tobago and the Institutional Review Board of The Scripps Research Institute, USA. All participants gave written informed consent before participating in the study.

A total of 144 alcohol dependent individuals of either East Indian or African ancestry were included in the study (i.e. participants with mixed ethnicity were excluded). Ethnicity was classified/ determined as having three grandparents from the same ethnic subgroup. Subjects with major medical problems that could possibly impact their drinking and were unrelated to

alcohol dependence (e.g. cancer, severe heart or lung disease, diabetes etc.) were excluded. The 
psychological consequences of medical conditions are well known, especially in situations where individuals lack appropriate coping mechanism and social support. The presence of medical conditions can therefore have a confounding effect on outcomes, with alcohol use being a secondary outcome. Patients were recruited from admissions to the three Substance Abuse Centres. One hundred and thirteen (113) unrelated subjects who were not alcohol dependent were matched by age, sex and ethnicity to the alcohol dependent participants. These controls were recruited through fliers distributed in the communities and by word of mouth.

\section{Psychiatric Assessment}

All participants were interviewed by one of three consultant psychiatrists (with postgraduate qualifications in psychiatry) using the Semi-Structured Assessment for the Genetics of Alcoholism (SSAGA) to determine a diagnosis of alcohol dependence or abuse. The SSAGA is an instrument designed to assess physical, psychological and social manifestations of alcoholism and related disorders. It was previously validated by the Collaborative Group on the Genetics of Alcoholism (COGA) in the USA (4). The SSAGA covers the major DSM-III-R psychiatric disorders, as well as anti-social personality disorder (ASPD). Psychiatric diagnoses were derived on the basis of computer algorithms for DSM-III-R diagnoses. This assessment confirms the presence of anti-social personality disorder with conduct disorder before the age of 15 but also identifies syndromal levels of anti-social behaviour after the age of 15 years.

In situations where the independent psychiatric disorder was likely due to a significant stressor in the participant's life (i.e. a medical illness, injury, a medication or toxin, the death of someone close to the participant - bereavement), psychiatric diagnoses of adjustment reaction were made. Psychiatric disorders due to the non-prescribed use of medication, such as benzodiazepines and 
opiates, were classified as substance induced. For each disorder, the percentage of participants, who had a lifetime history of the independent and/or the substance-induced types of the same disorder, was also calculated.

\section{Data analysis}

Comparative quantitative analysis between the alcohol-dependent subjects and the control subjects in the two ethnic groups was performed using ANOVA (Analysis of Variance), while analysis of categorical variables was performed using chi-square $\left(\chi^{2}\right)$ test or Fisher's exact test depending on cell size. In these analyses, a $p$-value $<0.05$ was considered statistically significant. The sample was divided into those with and without alcohol dependence and then in each subsample the presence (yes vs. no) of ASPD was separately compared to each demographic variable, using both continuous (ANOVA) and dichotomous (chi-square).

\section{RESULTS}

One hundred (100) East Indian- and forty four (44) African- ancestry subjects with a diagnosis of alcohol dependence participated in this study, and they were matched with sixty seven (67) East Indian- and forty six (46) African-ancestry controls respectively. There were no significant differences in gender and ethnicity, however there were significant differences in marital status and employment between the control group and the alcohol dependent group (Table 1). 
Table 1: Demographic characteristics of Alcohol dependent versus Control groups

\begin{tabular}{|c|c|c|c|}
\hline Characteristics & $\begin{array}{l}\text { Alcohol dependent } \\
(\mathrm{n}=144) \\
\mathrm{n}(\%)\end{array}$ & $\begin{array}{l}\text { Control }(n=113) \\
\text { n }(\%)\end{array}$ & p value \\
\hline \multicolumn{4}{|l|}{ Gender } \\
\hline Male & $129(89.6 \%)$ & $100(88.5 \%)$ & 0.841 \\
\hline Female & $15(10.4 \%)$ & $13(11.5 \%)$ & \\
\hline \multicolumn{4}{|l|}{ Ethnicity } \\
\hline East Indian Ancestry & $100(59.9 \%)$ & $67(40.1 \%)$ & 0.114 \\
\hline African Ancestry & $44(48.9 \%)$ & $46(51.1 \%)$ & \\
\hline \multicolumn{4}{|l|}{ Married } \\
\hline Yes & $51(35.4 \%)$ & $79(69.9 \%)$ & 0.000 \\
\hline No & $93(64.6 \%)$ & $34(30.1 \%)$ & \\
\hline \multicolumn{4}{|l|}{ Employed } \\
\hline Yes & $98(68.1 \%)$ & $107(94.7 \%)$ & 0.000 \\
\hline No & $46(31.9 \%)$ & $6(5.3 \%)$ & \\
\hline \multicolumn{4}{|l|}{ Employed fulltime } \\
\hline Yes & $80(57.1 \%)$ & $104(93.7 \%)$ & 0.000 \\
\hline No & $60(42.9 \%)$ & $7(6.3 \%)$ & \\
\hline
\end{tabular}

Statistically significant, $p<0.05$

All participants did not complete the section of the assessment tool about employment and whether it is full time.

Table 2 represents the analysis comparing the control group to the alcohol dependent group with focus on anti-social personality disorder. Seven (7) East-Indians and five (5) Africans met the diagnosis for $\mathrm{ASPD} / \mathrm{CD}$ from the alcohol dependent group, while two male controls met the diagnosis of ASPD, one from each of the two ethnic groups. The difference in ASPD/ CD between the alcohol dependent group and control group was significant $(p=0.026)$. 
Table 2: Comparison of ASPD \& CD in Alcohol dependent versus Control groups

\begin{tabular}{|c|c|c|c|}
\hline Diagnoses & $\begin{array}{l}\text { Alcohol dependent }(n=144) \\
\text { n }(\%)\end{array}$ & $\begin{array}{l}\text { Control }(n=113) \\
n(\%)\end{array}$ & p value \\
\hline \multicolumn{4}{|l|}{$A S P D / C D$} \\
\hline Yes & $12(8 \%)$ & $2(1.8 \%)$ & 0.026 \\
\hline No & $132(92 \%)$ & $111(98.2 \%)$ & \\
\hline \multicolumn{4}{|l|}{$A S P D$} \\
\hline Yes & $5(3.5 \%)$ & $2(1.8 \%)$ & 0.471 \\
\hline No & $139(96.5 \%)$ & $111(98.2 \%)$ & \\
\hline \multicolumn{4}{|l|}{$C D$} \\
\hline Yes & $7(5.0 \%)$ & $\mathbf{0}$ & 0.019 \\
\hline No & $137(95 \%)$ & $\mathbf{0}$ & \\
\hline
\end{tabular}

Table 3 shows the detection of ASPD/ CD compared by ethnic group. Statistically there were no significant differences in ASPD/ CD between individuals of East Indian and African ancestry with diagnoses of alcohol dependence

Table 3. Ethnic differences of ASPD/ CD in alcohol dependent subjects

\begin{tabular}{lccc}
\hline \multirow{3}{*}{ Diagnosis } & East Indian $(\mathrm{n}=\mathbf{1 0 0})$ & $\begin{array}{l}\text { African }(\mathrm{n}=44) \\
\mathrm{n}(\%)\end{array}$ & $\boldsymbol{p}$ value \\
& $\mathrm{n}(\%)$ & $5(11.4 \%)$ & 0.383 \\
\hline $\boldsymbol{A S P D / C D}$ & $7(7 \%)$ & $39(88.6)$ & \\
Yes & $93(93 \%)$ &
\end{tabular}

In the overall comparison of the two ethnic subgroups there were no significant differences in psychosocial characteristics of gender, marital and employment status when ASPD group were compared with non-ASPD in the control and alcohol dependent groups. 


\section{DISCUSSION}

This study was an attempt to examine the Trinidad and Tobago COGA study sample, comparing ASPD/ CD in the two major ethnic subgroups. The analysis showed significant differences in ASPD/ CD between alcohol dependent groups and control groups in Trinidad and Tobago. This compares with many other studies that reported higher rates of personality disorders in alcohol dependent groups compared to non-alcohol dependent groups $(8,9)$. Although, the sample under review is a treatment group with no general population data to compare, the findings are still consistent with those found in other studies (17).

In regard to ethnicity no significant differences in psychosocial characteristics (i.e. gender, marital and employment status) were detected when ASPD group was compared with non-ASPD in both control and alcohol dependent subjects. These findings may initially suggest that personality disorder may not play an important role in difference in alcohol dependence between these two major ethnic subgroups. However, the sample size may also explain the overall outcome and result therefore the result is non-generalisable. The significant differences in marital status and employment or full-time employment status between alcohol dependent and control subjects are predictable given the impaired functioning and coping skills demonstrated in the alcohol dependent individual.

The low percentage of personality disorder in alcohol-dependent subjects (East Indian $7 \%$ and Africans $11 \%$ ) from this sample varies significantly from findings from international studies (18) but supports previous findings (19) of reportedly low levels of personality disorder 
in the alcoholic population of this country. The finding is also in contrast to findings from other studies of outpatient clinics with alcohol use disorders that found a prevalence of personality disorder between 40\% and 64\% (20, 21). Sher and Trull (1994) determined that ASPD and alcoholism shared important indicators in the etiological models of these pathologies, although unspecific, thereby further associating them as co-morbidies (22). In fact some research suggests that the disposition of these mental disorders is ASPD followed by alcohol dependence, relegating the latter diagnosis as secondary (23) and thereby suggesting ASPD as an important co-factor in the development and severity of alcohol dependence. Other researchers (24) also detected similar associations but have been more cautious in making conclusions citing the need for further investigations. Providing more objective data was a meta-analysis in which ASPD and substance use disorders have significant co-existence possibly due to shared personality traits (25). Therefore elucidating these findings in this population may be determined by a larger, representative sample size to establish concurrence.

Research also suggests that statistically significant associations between $C D$ and alcohol use disorders are detected in women (26). Also in the US sample (27) a significant association was only found in women but in this present study sample no female met the criteria for a diagnosis of personality disorder. Overall, the sample consisted of a small number of participants that met the diagnosis for personality disorder using the DSM-III-R criteria. Though sample size may be constraint affecting the concurrence of some of the results of this study with other research in the field, other factors may provide similar hindrance. 
Throughout the Caribbean there remains a great amount of stigma surrounding the diagnosis of mental disorders. This stigma influences reporting attitude among the populations at large, leading to under-reporting of inappropriate and observable asocial behaviour. Mental illness and all the associated impairment is still attributed to "demons" and "spirits" by large sections of the population. Asocial behaviour in this context is 'culturally unacceptable behaviour' from individuals and groups. The perception that mental disorder is due to many unexplainable factors, influences the type of data that can be obtained from participants. Also, the social acceptability of a particular behaviour may be rooted in cultural belief that influences the type of information that will be provided by informants, e.g. Carnival and pre-Carnival events represent a time of excessive alcohol consumption that is socially sanctioned. The results of this study are further limited by design, a one-time cross sectional survey, which may be associated with under-estimations of lifetime prevalence of mental disorder (28). The cultural behaviour differences cannot be conclusively explained by the co-occurrence of personality disorder due to the lack of epidemiological data.

While the result may not be generalisable, there is implication for management in individuals with co-existing disorders, by encouraging appropriate screening for the presence or absence of personality disorder in identifiable populations. The findings so far provided inadequate explanation to accept the hypothesis that there are differences in personality disorder diagnosis in alcohol abuse/ dependent patients within this population and present no definite factors to explain the detectable differences. However, individual and societal attitudes and practices in festive and celebrative events have been partially blamed, in addition to other rearing practices (2). These attitudes include the high tolerance for alcohol consumption and its 
association with cultural events like carnival as well as little enforcement of age requirement for alcohol purchase and consumption.

Understanding the role of personality disorders on alcohol abuse/dependence comorbidity may highlight the possible role as a predictor for alcohol addiction in the Trinidad and Tobago population. Furthermore, approaches could be taken to address these disorders during early age as a preventive measure for alcoholism, especially if psychological factors play major role in alcohol abuse.

Similarly, early recognition of this co-morbidity may also allow proper interventional methods that are culturally designed to improve treatment outcome and reduce treatment relapse.

\section{Limitations}

The sample size of this study was insufficient to have external validity, therefore the current findings cannot be generalised to the two subpopulations investigated. The exclusion criteria used to select this sample population also restricts generalisation to the entire population of the country, since the excluded group consist about $20 \%$ of the population.

Resolution of these limitations will allow better comparability to the findings of international epidemiological studies that detected personality disorder among alcoholic patients in treatment settings or the general population.

The study design, a one-time cross sectional survey, relied on memory recall and the subjective responses from participants. This may result in inaccurate estimations of drinking behaviours and mental pathologies. 
Lifetime prevalence estimates have been noted to be susceptible to recall bias and other memory distortions (27). This may lead to underestimation or overestimation of true lifetime prevalence of mental disorders (29). Determinations of cultural behaviour differences and their impact on the co-occurrence of personality disorder are inconclusive due to the lack of the associated epidemiological data.

\section{CONCLUSIONS}

In the current study no significant differences in ASPD among alcohol dependent treatment subjects of East Indian and African ancestry in Trinidad and Tobago were detected. However, this study was done in an environment with intense negative view about mental illness. This negative view may influence the type of information that participants provide about their drinking behaviour. Drinking behaviours are sometimes socially acceptable, therefore may account for the low percentage of ASPD in the treatment group. The findings can however be used as a guide for future studies that will allow conclusive insights into the two subpopulations primarily selected, as well as the methodology for inclusion of the other ethnic subpopulations and finally generalisation to the entire population. Adding to the knowledge of the co-morbidity of substance disorders with antisocial syndromes will clarify implications for management and prevention.

\section{ACKNOWLEDGMENT}

We received contribution from David A. Guilder of Technical support: Data Analysis, Molecular and Integrative Neurosciences Department, The Scripps Research Institute, 10550 North Torey Pines Road, La Jolla, CA 92037 USA and Email: dgilder@scripps.edu. 


\section{AUTHOS' NOTE}

The research was supported by National Institute on Alcohol Abuse and Alcoholism grants AA006420 and AA014370, the Stein Endowment Fund, and the University of the West Indies. 


\section{REFERENCES}

1. American Psychiatric Association. Diagnostic and Statistical Manual of Mental Disorders - Version V. Washington, DC, USA, 2013.

2. Montane LK, Maharaj H. Ethnic differences in the incidence rates of mental disorders in country Caroni, Trinidad. West Indian Medical J. 1998: 48(Suppl 2): 50.

3. The Central Statistical Office: Trinidad and Tobago. Population and Housing Census Demographic Report. Port of Spain, Trinidad and Tobago, 2011. www.cso.planning.gov.tt/census. [Accessed March 5, 2015].

4. Hesselbrock MN, Easton C, Bucholz KK, Schuckit M, Hesselbrock VA. Validity of SSAGA - a comparison with the SCAN. Addiction 1999; 94: 1361-1370.

5. Ross HE, Glazer, FB, Germanson T. The prevalence of psychiatric disorders in patients with alcohol and other drug use problems. Arch. Gen. Psychiatry 1988; 45: 1023-1031.

6. Skodol AE, Oldham JM, Gallahar PE. Axis II Comorbidity of substance use disorders among patients referred for treatment of personality disorder. Am. J. Psychiatry 1999; 156: $733-738$.

7. Gilder DA, Lau P, Gross A, Ehlers CL. Co-morbidity of alcohol dependence with other psychiatric disorders in young adult Mexican Americans. J. Addict. Dis 2007; 26: 31-40.

8. Ladd GT, Petry NM. Antisocial personality in treatment-seeking cocaine abusers: psychosocial functioning and HIV risk. J. Subst. Abuse. Treat 2003; 24: 323-330. 
9. Goldstein RB, Dawson DA, Saha TD, Ruan WJ, Compton WM, Grant BF. Antisocial behavioural syndromes and DSM-IV alcohol use disorders: Results from the national epidemiologic survey on alcohol and related conditions. Alcoholism: Clinical and Experimental Research 2007; 31(5): 814-828.

10. Helzer JE, Pryzbeck TR. The co-occurrence of alcoholism with other psychiatric disorders in the general population and its impact on treatment. J. Stud Alcohol. 1988; 49: $219-224$

11. Hessselbrock, MN, Meyer RE, Keener JJ. Psychopathology in Hospitalized Alcoholics. Arch Gen Psychiatry 1985; 42: 1050-5.

12. Howard MO, Walker RD, Suchinsky RT, Anderson B. Substance-use and psychiatric disorders among American Indian veterans. Subs Use Misuse 1995; 31: 581-598.

13. Hesselbrock MN, Hesselbrock VM, Segal B, Schuckit MA, Bucholz K. Ethnicity and Psychiatric Comorbidity Among Alcohol-Dependent Persons Who Receive Inpatient Treatment: African Americans, Alaska natives, Caucasians, and Hispanics. Alcohol Clinical and Experimental Research 2003; 27(8): 1368-1373.

14. Beaubrun MH, Firth H. A Transcultural analysis of Alcoholics in Trinidad/London. Paper presented at Joint Meeting of the Caribbean Psychiatric Association and the American Psychiatric Association, Ocho Rios, Jamaica, May 10-14, 1969. [Unpublished].

15. Shafe S, Guilder DA, Montane-Jaime LK, Josephs R, Moore S, Crooks H, Ramcharan C, Ehlers CL. Co-morbidity of alcohol dependence and select affective and anxiety 
disorders among individuals of East Indian and African ancestry in Trinidad and Tobago. West Indian Med. J 2009; 58 (2): 164-172.

16. Hutchinson G. Substance and Mortality. UWI Today, St. Augustine, Trinidad and Tobago, 2012.

17. Langås AM, Malt UF, Opjordsmoen S. In-depth study of personality disorders in firstadmission patients with substance use disorders. BMC Psychiatry. 2012 Oct 29;12: 180. doi: 10.1186/1471-244X-12-180. Last accessed November 252015

18. Grant BF, Stinton FS, Dawson DA, Chou SP, Ruan WJ, Pickering RP. Co-Occurrence of 12-Month Alcohol and Drug Use Disorders and Personality Disorders in the United States. Alcohol Research and Health 2006; 29 (4): 121-130.

19. Beaubrun MH. The nature of alcohol dependence and its recognition. Medicine Digest 1982; 8(1): 5-13.

20. Nurnberg HG, Rifkin A, Doddi S. A systematic assessment of the comorbidity of DSMIII-R personality disorders in alcoholic outpatients. Compr Psychiatry 1993; 34: 447-454.

21. Echeburua E, De Medina RB, Aizpiri J: Alcoholism and personality disorders: an exploratory study. Alcohol Alcohol 2005; 40: 323-326.

22. Sher KJ, Trull TJ. Personality and dis-inhibitory psychopathology: alcoholism and antisocial personality disorder. J Abnorm. Psychol. 1994; 103(1):92-102.

23. Bahlmann M, Preuss UW, Soyka M. Chronological relationship between antisocial personality disorder and alcohol dependence. Eur. Addict Res. 2002; 8(4): 195-200. 
24. Alterman AI, Cacciola JS. The antisocial personality disorder diagnosis in substance abusers: problems and issues. J Nerv Ment Dis. 1991; 179(7): 401-9.

25. Ruiz MA, Pincus AL, Schinka JA. Externalizing pathology and the five-factor model: a meta-analysis of personality traits associated with antisocial personality disorder, substance use disorder, and their co-occurrence. J Pers Disord. 2008; 22(4): 365-88.]

26. Compton WM, Conway KP, Stinson FS, Colliver JD, Grant BF. Prevalence, correlates, and comorbidity of DSM-IV antisocial personality syndromes and alcohol and specific drug use disorders in the United States: results from the national epidemiologic survey on alcohol and related conditions. J Clin Psychiatry. 2005; 66(6): 677-85

27. Kessler RC, McGonagle KA, Zhao S. Lifetime and 12 months prevalence of DSM-111R psychiatric disorders in the United States. Arch Gen Psychiatry 1994; 51(1):8-19.

28. Takayanagi Y, Spira AP, Roth KB, Gallo JJ, Eaton WW, Mojtabai R. Accuracy of Reports of Lifetime Mental and Physical Disorders: Results from the Baltimore Epidemiological Catchment Area Study; JAMA Psychiatry. 2014; 71(3):273-280. Doi:1001/jamapsychiatry.. Last accessed December 2015.

29. Patten SB, Williams JU, Lavorato DH, Bulloch AG, D'Arcy C, Streiner DL. Recall of recent and more remote depressive episodes in a prospective cohort study. Soc Psychiatry Psychiatr Epidemiol. 2012; 47 (5): 691-696. 